\title{
Dinámica folicular durante el ciclo estral natural en búfalas (Bubalus bubalis)
}

\author{
Follicular dynamics of the natural estrous cycle in buffalo (Bubalus bubalis)
}

Jorge Alberto Sánchez ${ }^{1,2}$, Marlyn Hellen Romero', Audelo Fernando Meneses ${ }^{1}$

\section{Resumen}

El objetivo del estudio fue monitorear la dinámica folicular del ciclo estral natural de hembras bufalinas en Colombia. Se evaluaron los patrones de crecimiento y regresión de folículos ováricos de 11 búfalas durante dos ciclos estrales. Se realizaron exámenes ecográficos interdiarios de los ovarios para establecer patrones de ondas foliculares, número de ondas, día de emergencia, número de folículos reclutados, y características del folículo dominante (FD) y del cuerpo lúteo (CL). Las concentraciones de progesterona $\left(\mathrm{P}_{4}\right)$ se determinaron por radioinmunoensayo. Se evaluaron 17 ciclos normales $(2,11$ y 4 ciclos con una, dos y tres ondas foliculares, respectivamente). El intervalo inter-ovulatorio fue de $12.5 \pm 2.7,20.5 \pm 1.2$ y $28.8 \pm 1.9$ días para los ciclos con 1,2 y 3 ondas foliculares, respectivamente. Las ondas foliculares emergieron los días $0 \pm 2.3$ en ciclos de una onda, los días $2.3 \pm 1.0$ y $10.5 \pm 1.0$ en ciclos de dos ondas y los días $1.25 \pm 1.6,8.0 \pm 1.9$ y 20.8 \pm 1.6 (promedio \pm e.e.) en ciclos de tres ondas. El diámetro máximo $(\mathrm{cm})$ alcanzado por el FD fue de $1.2 \pm 0.4$ en ciclos de una onda, de $1.02 \pm 0.2$ y $1.3 \pm 0.3$ en ciclos de dos ondas, y de $1.2 \pm 0.3,1.3 \pm 0.3$ y $1.2 \pm 0.3$ en ciclos de tres ondas. La concentración de P4 en el día de máximo diámetro del CL en promedio fue de $7.2 \pm 6.6 \mathrm{ng} / \mathrm{ml}$. Se concluye que la dinámica folicular de la búfala colombiana se caracteriza por presentar patrones de una, dos y tres ondas foliculares, con predominio de ciclos de dos ondas.

Palabras clave: búfalos; ciclo estral; folículo; ondas foliculares; progesterona

\section{AbSTRACT}

The aim of the study was to monitor the follicular dynamics of the natural estrous cycle in the domestic buffalo in Colombia. Patterns of growth and regression of ovarian follicles in two consecutive oestrus cycles in 11 buffalos were evaluated. Ultrasound examination of the ovaries were conducted every second day to establish patterns of

\footnotetext{
${ }^{1}$ Laboratorio de Endocrinología y Reproducción Animal, Departamento de Salud Animal, Grupo CIENVET, Programa Medicina Veterinaria y Zootecnia, Facultad de Ciencias Agropecuarias, Universidad de Caldas, Manizales, Colombia

${ }^{2}$ Email: jorge.sanchez@ucaldas.edu.co

Recibido: 7 de mayo de 2018

Aceptado para publicación: 6 de diciembre de 2018
} 
follicular waves, number of waves, emergency day, number of follicles recruited, and characteristics of the dominant follicle (DF) and of the corpus luteum (CL). Progesterone $\left(\mathrm{P}_{4}\right)$ concentration was determined by radioimmunoassay. Seventeen normal cycles $(2,11$ and 4 estrous cycles with one, two and three follicular waves, respectively) were evaluated. The inter ovulatory interval was $12.5 \pm 2.7,20.5 \pm 1.2$ and $28.8 \pm 1.9$ days for cycles with 1,2 and 3 follicular waves, respectively. Follicular waves emerged on days $0 \pm 2.3$ for cycles with 1 wave, on days $2.3 \pm 1.0$ and $10.5 \pm 1.0$ in cycles with two waves and on days $1.6 \pm 1.25,8.0 \pm 1.9$ and $20.8 \pm 1.6$ (mean \pm e.e.) in cycles with the waves. The concentration of 44 on the day of maximum diameter of CL on average was $7.2 \pm 6.6 \mathrm{ng} / \mathrm{ml}$. In conclusion, follicular dynamics of the Colombian buffalo is characterized by patterns of one, two and three follicular waves, with predominance of two-wave cycles.

Key words: buffalo; estrous cycle; follicle; follicular wave; progesterone

\section{INTRODUCCIÓN}

La producción del búfalo doméstico (Bubalus bubalis) es de gran importancia en la economía agropecuaria de muchos países de Asia y América Latina. Estos animales están adaptados a climas subtropicales y tropicales, teniendo un gran potencial para la producción de trabajo, carne y leche, especialmente en suelos pobres de baja fertilidad (Singh et al., 2000; Chaikhun et al., 2010; Perera, 2011).

La reducida eficiencia reproductiva del búfalo afecta la productividad de la hembra, debido al inicio tardío de la pubertad, estacionalidad de nacimientos, pobre expresión del estro, largo anestro posparto y prolongado intervalo entre partos. Estas características pueden ser parcialmente atribuidas a cambios en la estacionalidad de lluvias, lo que produce una menor disponibilidad de alimentos(Murugavel et al., 2009; Nam, 2010; Sah y Nakao, 2010; Sanchez et al., 2017) y estrés térmico en el animal, caracterizado por elevadas secreciones de prolactina (Roy y Prakash, 2007). Además, se puede dar por cambios en el fotoperiodo y, por lo tanto, a alteraciones en la secreción de melatonina (Murugavel et al., 2009; Nam, 2010; Perera, 2011).
La aplicación de la inseminación artificial (IA) en el búfalo se ve afectada por la baja expresión del celo, siendo común el estro silente (Rajanarayanan y Archunan, 2011). La concentración de 17 $\beta$-estradiol durante el estro normal es más baja, comparada con la del bovino (Carvalho et al., 2002), por lo que se prefiere la IA a término fijo (IATF) para implementar programas de servicios en búfalos (Abdullah et al., 2001; Ali y Fahmmy, 2007). Asimismo, la presencia del macho durante el posparto favorece el reinicio de la actividad cíclica ovárica, reduce la incidencia de estros silentes y mejora la tasa de concepción al primer servicio (Gokuldas et al., 2010).

La duración del ciclo estral (CE) está relacionada con el número de ondas foliculares que se desarrollan en cada ciclo. No obstante, el desarrollo de folículos primordiales hasta folículos en crecimiento y folículos terciarios aparenta ser poco efectivo en la especie bufalina, pues se presenta un número mayor de folículos atrésicos con relación al bovino (Madan et al., 1996; Campanile et al., 2010; Gimenes et al., 2011; Baruselli et al., 2013). Los búfalos presentan cerca de 12000 folículos primarios al nacimiento, cifra bastante menor a los 133000 reportados para bovinos (Danell, 1987; Drost, 2007). 
Según datos del ICA (2015), Colombia cuenta con una población bufalina cercana a los 235000 animales distribuidos en 2672 predios, concentrados principalmente en los departamentos de Córdoba (24.8\%), Antioquia $(20.4 \%)$ y Santander $(12.6 \%)$, los cuales agrupan el $57.8 \%$ de la población nacional. Debido a que el búfalo presenta una alta capacidad de adaptación y rusticidad, se requiere estudiar su comportamiento reproductivo local para diseñar estrategias que mejoren el uso de biotecnologías y coadyuven a la mejora de su reproducción (Brito et al., 2002). En Colombia no se han realizado estudios completos sobre la fisiología reproductiva de la búfala, que faciliten la comprensión de la dinámica folicular y el comportamiento endocrino. El objetivo del presente estudio fue describir la dinámica folicular en el ciclo estral natural de las búfalas en el trópico colombiano bajo.

\section{MATERIALES y Métodos}

\section{Localización}

El estudio se realizó en la región del Magdalena Medio colombiano, en la hacienda La Gloria del municipio de Puerto Salgar, Colombia. El clima de la zona se caracteriza por ser un bosque húmedo tropical, con temperatura promedio de $26{ }^{\circ} \mathrm{C}$, pluviosidad de $1420 \mathrm{~mm}$ y una altura de $150 \mathrm{msnm}$. El experimento se llevó a cabo durante la estación de verano (junio-noviembre) de 2013.

\section{Animales}

Se seleccionaron 20 hembras bufalinas raza Trinitaria (búfalo colombiano) del total del hato, de 6 años de edad, con peso corporal promedio de $450 \mathrm{~kg}$ y condición corporal de 3.5 dentro de una escala de 1-5 (Alapati et al., 2010), con intervalo posparto entre 60 y 150 días y con cría al pie. Los animales no estaban bajo ningún protocolo de sincronización para poder evaluar el ciclo estral de forma natural.
Las búfalas tenían tres lactancias en promedio y estuvieron acompañadas por un butoro recelador para determinar el comportamiento de estro. Los animales se observaron cada 12 horas (06:00 y 18:00) durante dos ciclos consecutivos. El examen fue realizado por un único operador, médico veterinario especialista en reproducción bovina. Las búfalas fueron consideradas en estro cuando aceptaban ser montadas por el toro recelador, presentaban descarga de moco cristalino por la vulva o el dispositivo Kamar ${ }^{\circledR}$ activado. Todas las vacas recibieron el mismo manejo sanitario y fueron mantenidas en pastoreo extensivo en potreros con pasto Angleton (Dichanthium aristatum), sal mineralizada al $8 \%$ y agua a voluntad. Los potreros contaban con zonas con sombra y cuerpos de agua para las actividades de termorregulación.

\section{Examen Ecográfico}

Una vez detectado el estro, se evaluó mediante ecografía la tonicidad del útero, el flujo vaginal y las estructuras ováricas, teniendo en cuenta el grado de ecogenicidad del endometrio y la delimitación marcada del endometrio y el miometrio, además del acúmulo de líquido en el lumen uterino (Descôteaux et al., 2010).

Se utilizó un ecógrafo veterinario Aquila VetPro ${ }^{\circledR}$ (Esaote SpA, Pie Medical, Países Bajos), provisto de una sonda rectal de 6-8 Mhz. Se registraron y midieron los folículos con diámetro mayor o igual a $0.3 \mathrm{~cm}$. Los ovarios fueron monitoreados cada dos días por dos o tres ciclos estrales consecutivos para establecer el intervalo inter-ovulatorio (IIO), el folículo dominante (FD), catalogado como mayor de $1 \mathrm{~cm}$ de diámetro y el mayor de los folículos presentes. Los folículos subordinados (FS) (cohorte folicular) fueron aquellos que aparecieron juntamente con el pool folicular del FD, donde un FD y su cohorte se definió como una onda folicular. El día de emergencia de la onda folicular se estableció como el día de la primera observación de un folículo que alcanzó $\operatorname{los} 0.3 \mathrm{~cm}$ de diámetro. 
Además, se determinó la vida útil del FD (días entre su aparición y su atresia completa), el máximo diámetro (promedio de los diámetros máximos de cada folículo), el día donde alcanzó su mayor diámetro y la tasa de crecimiento (máximo diámetro del folículo dominante dividido por la duración del crecimiento). El cuerpo lúteo (CL) se fijó como estructura poco ecogénica derivado del FD (Barkawi et al., 2009). Se tuvo la precaución de identificar CL originados por luteinización de folículos no ovulatorios y excluirlos del estudio. En el tercer celo se realizó la IA y el diagnóstico de preñez se realizó por ecografía el día 26 después de la inseminación.

\section{Determinación de Progesterona}

Se recolectaron $10 \mathrm{ml}$ de sangre por venopunción yugular en un tubo Vacutainer ${ }^{\circledR}$ sin anticoagulante cada 48 horas durante dos ciclos estrales consecutivos. El suero fue separado por centrifugación durante $10 \mathrm{~min}$ a $3000 \mathrm{~g}$ y fue almacenado en tubos Ependorff ${ }^{\circledR}$ a $-20{ }^{\circ} \mathrm{C}$.

Los niveles de progesterona $\left(\mathrm{P}_{4}\right)$ se determinaron mediante el método de radioinmunoensayo en fase sólida (RIA), utilizando el kit RIA-DPC (Laboratorio Siemens, Munich, Alemania), con una sensibilidad de detección del 94-96\%. El análisis se corrió completo (el máximo de muestras), empleando una curva estándar al inicio y otra al final del ensayo. Se usaron controles intraensayo cada 12 muestras.

\section{Diseño Estadístico}

Los análisis se realizaron utilizando el paquete estadístico SAS 9.2 para Windows (SAS Institute Inc, North Carolina, EEUU). Se trabajó con dos grupos de datos: el primero para caracterizar las ondas foliculares y el FD, y el segundo para describir el CL y las concentraciones de $\mathrm{P}_{4}$.
Se realizó una comparación con las medias de cuadrados mínimos de las características de las ondas mediante un modelo lineal generalizado. Se utilizó el análisis de varianza para determinar el efecto entre las ondas y las variables independientes (duración del IIO, duración de la onda, día de emergencia de la onda, número de folículos reclutados menores de $0.3 \mathrm{~cm}$, número de folículos reclutados de 0.3 a $0.6 \mathrm{~cm}$, máximo diámetro, día de máximo diámetro, vida promedio y tasa de crecimiento del folículo dominante). Los resultados se presentaron como promedios y errores estándar (EE). Las diferencias entre las ondas se evaluaron con una prueba de comparación múltiple ajustada con Tukey-Kramer.

Se empleó una regresión lineal simple para analizar la relación entre las concentraciones de $\mathrm{P}_{4}$ y las variables independientes (diámetro máximo, día de máximo diámetro, vida promedio, tasa de crecimiento para el CL y preñez). En todos los análisis se consideró como significativo un valor de $\mathrm{p}<0.05$.

\section{Resultados}

De los 20 animales seleccionados, se descartaron nueve búfalas por aciclia o ciclos irregulares. Se evaluaron 21 ciclos estrales de los cuales 17 fueron catalogados como ciclos estrales de duración esperada y $4(19 \%)$ como anovulatorios, los cuales no fueron utilizados en el estudio.

La duración del IIO fue de $22.7 \pm 1.0$ días. El diámetro máximo del FD fue de 1.21 $\pm 0.07 \mathrm{~cm}$, alcanzado en el día $12.1 \pm 1.4$, con una vida promedio $10.1 \pm 0.6$ días y una tasa de crecimiento de $0.1 \pm 0.01 \mathrm{~cm} /$ día.

Las búfalas mostraron tres tipos de patrones foliculares: 2 ciclos de una onda $(9.5 \%)$, 11 ciclos de dos ondas (52.4\%) y 4 ciclos de tres ondas (19.0\%). A los animales que se les evaluaron tres ciclos consecutivos, el pri- 
Cuadro 1. Características del ciclo estral y las ondas foliculares en búfalas colombianas (11 hembras, 17 ciclos estrales)

\begin{tabular}{|c|c|c|c|c|}
\hline \multirow[b]{2}{*}{ Variables } & \multirow[b]{2}{*}{$\begin{array}{l}\mathrm{N} .^{\circ} \text { de } \\
\text { onda }\end{array}$} & \multicolumn{3}{|c|}{ Ondas foliculares por ciclo estral } \\
\hline & & $\begin{array}{l}\text { Una } \\
(\mathrm{n}=2)\end{array}$ & $\begin{array}{c}\text { Dos } \\
(\mathrm{n}=8)\end{array}$ & $\begin{array}{c}\text { Tres } \\
(\mathrm{n}=4)\end{array}$ \\
\hline Intervalo inter-ovulatorio (días) & & $12.5 \pm 2.7^{\mathrm{a}}$ & $20.5 \pm 1.2^{\mathrm{a}}$ & $28.8 \pm 1.9^{\mathrm{b}}$ \\
\hline \multirow{3}{*}{$\begin{array}{l}\text { Duración de la onda folicular } \\
\text { (días) }\end{array}$} & 1 & $11.5 \pm 2.8$ & $10.2 \pm 1.2$ & $8.5 \pm 2.0$ \\
\hline & 2 & - & $10.1 \pm 1.2$ & $10.7 \pm 2.3$ \\
\hline & 3 & - & - & $10.8 \pm 2.0$ \\
\hline \multirow{3}{*}{$\begin{array}{l}\text { Emergencia de la onda folicular } \\
\text { (días) }\end{array}$} & 1 & $0 \pm 2.3$ & $2.27 \pm 1.0^{\mathrm{A}}$ & $1.3 \pm 1.6^{\mathrm{A}}$ \\
\hline & 2 & - & $10.5 \pm 1.0^{\mathrm{B}}$ & $8.0 \pm 1.9^{\mathrm{A}}$ \\
\hline & 3 & - & - & $20.8 \pm 1.6^{\mathrm{B}}$ \\
\hline \multirow{3}{*}{$\begin{array}{l}\mathrm{N} .^{\circ} \text { de folículos }<0.3 \mathrm{~cm} \\
\text { reclutados por onda folicular }\end{array}$} & 1 & $5.5 \pm 4.0$ & $5.0 \pm 1.7$ & $9.0 \pm 2.8$ \\
\hline & 2 & - & $5.4 \pm 1.7$ & $0.7 \pm 3.2$ \\
\hline & 3 & - & - & $3.3 \pm 2.8$ \\
\hline \multirow{3}{*}{$\begin{array}{l}\mathrm{N} . \\
\text { reclutados folículos de } 0.3-0.6 \mathrm{~cm} \\
\end{array}$} & 1 & $1.0 \pm 1.3$ & $1.2 \pm 0.6$ & $0.5 \pm 0.9$ \\
\hline & 2 & - & $1.2 \pm 0.6$ & $1.0 \pm 1.1$ \\
\hline & 3 & - & - & $1.0 \pm 0.9$ \\
\hline
\end{tabular}

Los valores se presentan como media \pm error estándar. Las medias con diferente letra $(a, b)$ dentro de filas y dentro de columnas por variable $(A, B)$ indican diferencia estadística $(p<0.05)$.

mer ciclo exhibió patrones de una onda folicular, seguidos de ciclos con patrones de dos ondas foliculares, sin embargo algunas búfalas con ciclos estrales largos solo presentaron dos ciclos en el mismo seguimiento.

\section{Ondas Foliculares}

Se observó diferencia significativa en la duración del IIO $(\mathrm{p}<0.05)$, siendo de mayor duración en animales que presentaron un patrón de tres ondas ( $28.8 \pm 1.9$ días), así como en el día de la emergencia de las ondas. De otra parte, no se observaron diferencias significativas en el número de folículos reclutados por onda folicular (Cuadro 1).

\section{Características del Folículo Dominante}

El diámetro máximo de los FD y los días de ocurrencia, así como su tasa de crecimiento según las ondas foliculares se presentan en el Cuadro 2. No se encontraron diferencias significativas entre ondas con relación a las variables en estudio. Por otro lado, el coeficiente de Pearson demostró una asociación entre la duración del intervalo inter-ovulatorio y el número de ondas $(\mathrm{r}=0.797 ; \mathrm{p}<0.05)$.

\section{Cuerpo Lúteo y $\mathbf{P}_{4}$}

El CL presentó un tamaño promedio de $1.6 \pm 0.2 \mathrm{~cm}$ en el día $7.0 \pm 1.9$, una vida promedio de $10.9 \pm 2.3$ días y una tasa de crecimiento de $0.04 \pm 0.13 \mathrm{~cm} /$ día. La concentración de $\mathrm{P}_{4}$ en el día de máximo diámetro del CL en promedio fue de $7.2 \pm 6.6 \mathrm{ng} / \mathrm{mL}$.

La tasa de preñez en el ciclo estral natural fue de $36.3 \%$. Las concentraciones de $\mathrm{P}_{4}$ al día de la emergencia de la onda del folículo ovulatorio fue de $4.46 \pm 1.50 \mathrm{ng} / \mathrm{ml}$ en búfalas preñadas y de $1.96 \pm 1.23 \mathrm{ng} / \mathrm{ml}$ en búfalas vacías. Las concentraciones de $\mathrm{P}_{4}$ al 
Cuadro 2. Características del folículo dominante durante el ciclo estral natural en búfalas colombianas (11 hembras, 17 ciclos estrales)

\begin{tabular}{lcccc}
\hline \multirow{2}{*}{$\begin{array}{l}\text { Características del } \\
\text { folículo dominante (FD) }\end{array}$} & $\begin{array}{c}\mathrm{N}^{\circ}{ }^{\circ} \text { de } \\
\text { onda }\end{array}$ & & \multicolumn{3}{c}{ Ondas foliculares por ciclo estral } \\
\cline { 3 - 5 } & 1 & $1.2 \pm 0.4$ & $1.0 \pm 0.2$ & $1.2 \pm 0.3$ \\
& 2 & - & $1.3 \pm 0.2$ & $1.3 \pm 0.3$ \\
Diámetro máximo (cm) & 3 & - & - & $1.2 \pm 0.3$ \\
& 1 & $5.0 \pm 3.1$ & $4.9 \pm 1.3$ & $5.5 \pm 2.2$ \\
Día del diámetro máximo & 2 & - & $17.6 \pm 1.3$ & $15.7 \pm 2.5$ \\
& 3 & - & - & $24.0 \pm 2.2$ \\
& 1 & $11.5 \pm 2.8$ & $10.2 \pm 1.2$ & $8.5 \pm 2.0$ \\
Vida promedio (días) & 2 & - & $10.1 \pm 1.2$ & $10.6 \pm 2.3$ \\
& 3 & - & - & $10.7 \pm 2.0$ \\
& 1 & $0.1 \pm 0.0$ & $0.1 \pm 0.0$ & $0.1 \pm 0.0$ \\
Tasa de crecimiento & 2 & - & $0.1 \pm 0.0$ & $0.1 \pm 0.0$ \\
(cm/día) & 3 & - & - & $0.07 \pm 0.02$ \\
\hline
\end{tabular}

Los valores se presentan como media \pm error estándar. No hubo diferencias estadísticas entre medias

día de máximo diámetro del FD fue de $8.16 \pm$ $3.19 \mathrm{ng} / \mathrm{ml}$ en búfalas preñadas y de $2.97 \pm$ $2.61 \mathrm{ng} / \mathrm{ml}$ en búfalas vacias. El máximo tamaño del CL en búfalas preñadas fue de 1.63 $\pm 0.35 \mathrm{~cm}$ el día $6.0 \pm 3.23$ y en búfalas vacías de $1.54 \pm 0.26 \mathrm{~cm}$ el día $7.5 \pm 2.44$.

Las regresiones lineales mostraron asociación entre las concentraciones de $\mathrm{P}_{4}$ con el estado de preñez $\left(\mathrm{R}^{2}=0.62 ; \mathrm{p}<0.001\right)$ y la duración del ciclo estral $\left(\mathrm{R}^{2}=0.84 ; \mathrm{p}<0.001\right)$. Por otro lado, no se observaron asociaciones entre las concentraciones de $\mathrm{P}_{4}$ al máximo diámetro del FD, la vida promedio del FD y del CL ni las tasas de crecimiento del CL, ni entre el tamaño del CL y el tamaño del FD.

\section{Discusión}

La literatura científica señala que el reducido número de folículos en la superficie ovárica de la búfala se atribuye al mayor porcentaje de atresia folicular y a la escasa interrelación de las células de la granulosa, cuando se compara con el bovino (Feranil et al., 2004; Mondadori et al., 2007; Mondadori et al., 2010).

Los búfalos son animales poliéstricos estacionales (Baruselli et al., 1997; Nam, 2010). En general se reporta una duración del ciclo estral de 16 a 33 días y un IIO de 18 a 24 días (Baruselli et al., 1997; Barkawi et al., 2009; Nam, 2010; Perera, 2011), datos que concuerdan con lo observado en el presente estudio, en el cual se encontró una duración del IIO de $22.7 \pm 1.0$ días.

Los resultados de este estudio revelan que las búfalas presentas un predominio de dos $(52.4 \%)$ y tres ondas $(19.0 \%)$ foliculares en el ciclo estral, en concordancia con otros autores que describen un patrón de crecimiento folicular de dos ondas en el 63 al 83\% de los casos (Singh et al., 2000; Ali et al., 2003; De Renis y Lopez-Gatius, 2007; Azawi et al., 2009; Barkawi et al., 2009), seguido por pa- 
trones de tres ondas (25 a 33\%) y $3.3 \%$ de una onda folicular (Manik et al., 2002; Awasthi et al., 2006; Warriach y Ahmad, 2007; Campanile et al., 2010; Yindee et al., 2010).

En el estudio se evidenció en los animales a los cuales se les hizo el seguimiento de tres ciclos, que el primer ciclo exhibía patrones de una onda folicular, seguidos de ciclos con patrones de dos ondas foliculares. Este resultado contradice lo reportado por Taneja et al. (1996), quienes evaluaron búfalas durante dos ciclos, presentando dos ondas en el primero y una onda en el segundo ciclo estral, lo que corresponde a una alteración en el patrón de ondas que no ha sido reportada para los bovinos. No obstante, se sugiere que si las búfalas están en compañía del toro bufalino, los ciclos ovulatorios cortos de una onda (1517 días) ocurren con mayor frecuencia, siendo un patrón normal de la dinámica folicular que favorece las tasas de concepción (Abdalla, 2003).

Las diferencias significativas en la duración del IIO por efecto del número de ondas foliculares encontradas en el presente estudio $(\mathrm{p}<0.05)$, difieren de los promedios reportados por Taneja et al. (1996) de $23.0 \pm$ 1.0 vs. $21.3 \pm 0.9$ días de duración del IIO con una dinámica folicular de una y dos ondas, respectivamente, así como del estudio de Awasthi et al. (2006) quienes reportaron $20.8 \pm 1.0$ y $22.3 \pm 0.9$ días en animales con una dinámica folicular de una y dos ondas, respectivamente.

Barkawi et al. (2009) observaron diferencias significativas en el día de emergencia de la onda y en el número de folículos reclutados en búfalas egipcias, indicando que en los ciclos de tres ondas, la emergencia de la primera onda es más temprana y el número de folículos reclutados es mayor, comparados con patrones de dos ondas. Estos resultados solo coinciden con los del presente estudio en relación con el día de emergencia de la primera onda folicular, pues no se en- contró diferencias significativas en el número de folículos reclutados por onda. En la fase de selección, Baruselli et al. (1997) reportaron un promedio de 6 a 8 folículos reclutados por cada onda, resultado similar a este estudio donde se hallaron entre 5 a 6 folículos menores de $0.3 \mathrm{~cm}$.

En el presente estudio no se encontró diferencia en la tasa de crecimiento $(\mathrm{p}=0.615)$ ni en el diámetro máximo del folículo dominante ( $\mathrm{p}=0.788$ ), resultados que coinciden con los hallados por Presicce et al. (2005); sin embargo, Baruselli et al. (1997) reportaron diferencias en el diámetro máximo del primer folículo dominante $(15.0 \pm 2.3$ vs. $11.9 \pm$ $1.7 \mathrm{~mm}$ ) y el diámetro del folículo ovulatorio $(15.5 \pm 1.6$ vs. $13.4 \pm 1.3 \mathrm{~mm})$ registrados en la segunda y tercera onda folicular.

El promedio de las concentraciones de $\mathrm{P}_{4}$ del presente estudio concuerdan con valores reportados por Vale y Ribeiro (2005) y Mondal et al (2006, 2009), quienes presen$\tan$ niveles de $\mathrm{P} 4$ en día del estro de 0.3-0.6 $\mathrm{ng} / \mathrm{ml}$, llegando a niveles de $1.36-3.6 \mathrm{ng} / \mathrm{ml}$ en los días 13-13 para caer de nuevo a niveles basales el día 17 del ciclo estral.

\section{Conclusiones}

La dinámica folicular de la búfala bajo las condiciones del presente estudio se caracterizó por presentar patrones de una, dos y tres ondas foliculares, donde la duración del intervalo entre ovulaciones fue dependiente del número de ondas foliculares.

\section{Agradecimiento}

Fuente de financiación: Patrimonio autónomo Fondo Nacional de Financiamiento para la Ciencia, la Tecnología y la Innovación, Francisco José de Caldas-Colciencias, la Vicerrectoría de Investigaciones y Posgrados de la Universidad de Caldas y el Fondo Bufalero del Centro. 


\section{Literatura Citada}

1. Abdalla EB. 2003. Improving the reproductive performance of Egyptian buffalo cows by changing the management system. Anim Reprod Sci 75: 1-8. doi: 10.1016/S0378-4320(02)-00225-7

2. Abdullah $P$, Williamson NB, Parkinson TJ, Fathalla M. 2001. Comparison of estrus synchronization programmes in dairy cattle using oestradiol benzoate, short-acting progesterone and cloprostenol, or buserelin and cloprostenol. New Zeal Vet J 49: 201210. doi: 10.1080/00480169.2001.36233

3. Alapati A, Rao SR, Jeepalyam S, Patrapalle SM, Yemireddy KR. 2010. Development of the body condition score system in Murrah buffaloes: validation through ultrasonic assessment of body fat reserves. J Vet Sci 11: 1-8. doi: 10.4142/jvs.2010.11.1.1

4. Ali A, Abdel-Razek AK, Abdel-Ghaffar S, Glatzel PS. 2003. Ovarian follicular dynamics in buffalo cows (Bubalus bubalis). Reprod Domest Anim 38: 214218. doi: 10.1046/j.1439-0531.2003.00428.x

5. Ali A, Fahmy S. 2007. Ovarian dynamics and milk progesterone concentrations in cycling and non-cycling buffalo-cows (Bubalus bubalis) during Ovsynch program. Theriogenology 68: 23-28. doi: 10.1016/j.theriogenology.2007.03.011

6. Awasthi MK, Khare A, Kavani FS, Siddiquee GM, Panchal MT, Shah RR. 2006. Is one-wave follicular growth during the estrous cycle a usual phenomenon in water buffaloes (Bubalus bubalis). Anim Reprod Sci 92: 241-253. doi: 10.1016/j.anireprosci.-2005.05.024

7. Azawi OI, Ali AJ, Noaman UT. 2009. A study on the ovarian follicular dynamic in Iraqi Northern Buffaloes. Trop Anim Health Pro 41: 79-83. doi: 10.1007/ s11250-008-9156-Z
8. Barkawi AH, Hafez YM, Ibrahim SA, Ashour G, El-Asheeri AK, Ghanem N. 2009. Characteristics of ovarian follicular dynamics throughout the estrous cycle of Egyptian buffaloes. Anim Reprod Sci 110: 326-334. doi: 10.1016/j.anireprosci.2008.02.016

9. Baruselli PS, Mucciolo RG, Visintin JA, Viana WG, Arruda RP, Madureira EH, Oliveira CA, et al. 1997. Ovarian follicular dynamics during the estrous cycle in buffalo (Bubalus bubalis). Theriogenology 47: 1531-1547. doi: 10.1016/S0093-691X(97)00159-3

10. Baruselli P, Soares J, Gimenes L, Carvalho NA. 2013. Control of buffalo follicular dynamics for artificial insemination, superovulation and in vitro embryo production. Buffalo Bull 32: 160-176.

11. Brito LF, Satrapa $R$, Marson EP, Kastelic JP. 2002. Efficacy of PGF(2alpha) to synchronize estrus in water buffalo cows (Bubalus bubalis) is dependent upon plasma progesterone concentration, corpus luteum size and ovarian follicular status before treatment. Anim Reprod Sci 73: 23-35.

12. Campanile G, Baruselli PS, Neglia G, Vecchio D, Gasparrini B, Gimenes $L U$, Zicarelli L, et al. 2010. Ovarian function in the buffalo and implications for embryo development and assisted reproduction. Anim Reprod Sci 121: 111. doi: 10.1016/j.anireprosci.2010.03.012

13. Carvalho NAT, Baruselli PS, Zicarelli $L$, Madureira EH, Visintin JA. D'Occhio MJ. 2002. Control of ovulation with a GnRH agonist after superstimulation of follicular growth in buffalo: fertilization and embryo recovery. Theriogenology 58: 1641-1650. doi: 10.1016/S0093-691X(02)01057-9

14. Chaikhun T, Tharasanit T, Rattanatep J, De Rensis F, Techakumphu M. 2010. Fertility of swamp buffalo following the synchronization of ovulation by the sequential administration of $\mathrm{GnRH}$ and PGF2alpha combined with fixed- 
timed artificial insemination. Theriogenology 74: 1371-1376. doi: 10.1016/ j.theriogenology.2010.06.007

15. Danell B. 1987. Oestrous behavior, ovarian morphology and cyclical variation in follicular system and endocrine pattern in water buffalo heifers. PhD Thesis. Uppsala, Sweden: Swedish University of Agricultural Sciences. 94 p.

16. De Rensis F, Lopez-Gatius F. 2007. Protocols for synchronizing estrus and ovulation in buffalo (Bubalus bubalis): a review. Theriogenology 67: 209-216. doi: 10.1016/j.therioge-nology.2006.-09.039

17. DesCôteaux L, Colloton J, Gnemmi G 2010. Practical atlas of ruminant and camelid reproductive ultrasonography. Iowa, USA: Wiley-Blackwell. 244 p.

18. Drost M. 2007. Advanced reproductive technology in the water buffalo. Theriogenology 68: 450-453. doi: 10.1016/j.theriogenology.2007.04.013

19. Feranil JB, Isobe N, Nakao T. 2004. Cell proliferation in the atretic follicles of buffalo and cattle ovary. Reprod Domest Anim 39: 405-409. doi: 10.1111/ j.1439-0531.2004.00543.x

20. Gimenes LU, Carvalho NA, Sá Filho MF, Vannucci FS, Torres-Júnior JR, Ayres H, Ferreira RM, et al. 2011. Ultrasonographic and endocrine aspects of follicle deviation, and acquisition of ovulatory capacity in buffalo (Bubalus bubalis) heifers. Anim Reprod Sci 123: 175-179. doi: 10.1016/j.anire-prosci.2010.12.004

21. Gokuldas PP, Yadav MC, Kumar H, Singh G, Mahmood S, Tomar AK. 2010. Resumption of ovarian cyclicity and fertility response in bull-exposed postpartum buffaloes. Anim Reprod Sci 121: 236-241. doi: 10.1016/j.anireprosci.2010.06.005

22. [ICA] Instituto Colombiano Agropecuario. 2015. Colombia: Censo pecuario nacional - 2015. [Internet]. Disponible en: http://www.ica.gov.co/getdoc/ 8232c0e5-be97-42bd-b07b-9cdbfb$07 \mathrm{fcac} /$ Censos-2008.aspx
23. Madan ML, Das SK, Palta P. 1996. Application of reproductive technology to buffaloes. Anim Reprod Sci 42: 299-306. doi: 10.1016/0378-4320(96)01534-5

24. Manik RS, Palta P, Singla SK, Sharma V. 2002. Folliculogenesis in buffalo (Bubalus bubalis): a review. Reprod Fert Develop 14: 315-325. doi: 10.1071/ RD01126

25. Mondadori RG, Luque MCA, Santin TR, Báo SN. 2007. Ultrastructural and morphometric characterization of buffalo (Bubalus bubalis) ovarian preantral follicles. Anim Reprod Sci 97: 323-333. doi: 10.1016/j.anireprosci.2006.02.010

26. Mondadori RG, Santin TR, Fidelis AAG, Porfírio EP, Báo SN. 2010. Buffalo (Bubalus bubalis) pre-antral follicle population and ultrastructural characterization of antral follicle oocyte. Reprod Domest Anim 45: 33-37. doi: 10.1111/j.1439-0531.2008.01199.x

27. Mondal S, Prakash BS, Palta P. 2007. Endocrine aspects of oestrous cycle in buffaloes (Bubalus bubalis): an overview. Asian Austral J Anim 20: 124131. doi: 10.5713/ajas.2007.124

28. Mondal S, Suresh KP, Nandi S. 2009. Endocrine profiles of oestrous cycle in buffalo: a meta-analysis. Asian Austral J Anim 23: 169-174. doi: 10.5713/ ajas.2010.90193

29. Murugavel K, Antoine D, Raju MS, López-Gatius F. 2009. The effect of addition of equine chorionic gonadotropin to a progesterone-based estrous synchronization protocol in buffaloes (Bubalus bubalis) under tropical conditions. Theriogenology 71: 1120-1126. doi: 10.1016/j.theriogenology.2008.12.012

30. Nam NH. 2010. Characteristics of reproduction of the water buffalo and techniques used to improve their reproductive performance. J Sci Dev 8: 100-110.

31. Perera BM. 2011. Reproductive cycles of buffalo. Anim Reprod Sci 124: 194199. doi: 10.1016/j.anireprosci.2010.08 .022 
32. Presicce GA, Bella A, Terzano GM, De Santis G, Senatore EM. 2005. Postpartum ovarian follicular dynamics in primiparous and pluriparous Mediterranean Italian buffaloes (Bubalus bubalis). Theriogenology 63: 1430-1439. doi: 10.1016/j.theriogeno-logy.2004.07.003

33. Rajanarayanan S, Archunan G 2011. Identification of urinary sex pheromones in female buffaloes and their influence on bull reproductive behaviour. Res Vet Sci 91: 301-305. doi: 10.1016/j.rvsc.2010.12.005

34. Roy KS, Prakash BS. 2007. Seasonal variation and circadian rhythmicity of the prolactin profile during the summer months in repeat-breeding Murrah buffalo heifers. Reprod Fert Develop 19: 569-575. doi: 10.1071/RD06093

35. Sánchez JA, Romero MH, Suarez YJ. 2017. Estacionalidad reproductiva de la hembra bufalina (Bubalus bubalis). Rev Inv Vet Peru 28: 606-618. doi: 10.15381/rivep.v28i3.13289

36. Sah SK, Nakao TA. 2010. A clinical study of anestrus buffaloes in southern Nepal. J Reprod Develop 56: 208-211. doi: 10.1262/jrd.09-137T
37. Singh J, Nanda AS, Adams GP. 2000. The reproductive pattern and efficiency of female buffaloes. Anim Reprod Sci 60-61: 593-604. doi: 10.1016/S03784320(00)00109-3

38. Taneja M, Ali A, Singh G. 1996. Ovarian follicular dynamics in water buffalo. Theriogenology 46: 121-130. doi: 10.1016/0093-691X(96)00147-1

39. Vale WG, Ribeiro H. 2005. Características reprodutivas dos bubalinos: puberdade, ciclo estral, involução uterina e atividade ovariana no pós-parto. Rev Bras Reprod Anim 29: 63-73.

40. Warriach HM, Ahmad N. 2007. Follicular waves during the oestrous cycle in Nili-Ravi buffaloes undergoing spontaneous and PGF2alpha-induced luteolysis. Anim Reprod Sci 101: 332337. doi: 10.1016/j.anire-prosci.2007.01.013

41. Yindee M, Techakumphu M, Lohachit C, Sirivaidyapong S, Na-Chiangmai A, Colenbrander B. 2010. Ovarian activity and sexual behavior in the postpartum swamp buffalo (Bubalus bubalis). Ital J Anim Sci 6: 632-635. doi: 10.4081/ijas.2007.s2.632 